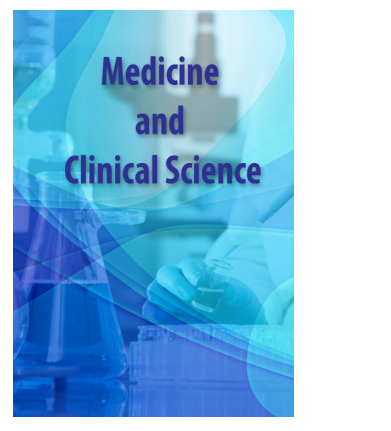

Correspondence

Ralph Pelligra, M.D.

52 Liberty Dock, Sausalito, CA. 94965, Tel: 415-272-1314.

E-mail: ralph.Pelligra-1@nasa.gov

- Received Date: 03 Apr 2020;

- Accepted Date: 26 Apr 2020;

- Publication Date: 01 May 2020.

Keywords

Seizures, Modern science, Sleep, Brain, Rest.

Copyright

(C) 2020 Science Excel. This is an openaccess article distributed under the terms of the Creative Commons Attribution 4.0 International license.

\title{
Metabolic Correlates of Sleep and Seizures: The Basis for a Unified Concept of Seizure Onset and Termination
}

Ralph Pelligra, M.D.

The Institutes for the Achievement of Human Potential (IAHP) 8801 Stenton Avenue, Wyndmoor, PA 19038, US.

\begin{abstract}
Two questions of fundamental clinical importance in epilepsy research continue to resist scientific inquiry: when and why does a seizure begin; and when and why does a seizure end?

The answers may be embedded in the counter-intuitive relationship between sleep and seizures. Deep sleep (NREM) and seizures, seemingly disparate conditions, share two distinctive features: altered consciousness, and electroencephalograph (EEG) slow waves of synchronized neuronal activity. Both altered consciousness and EEG slow waves are induced by elevated levels of adenosine, a breakdown product of the biological energy source, adenosine triphosphate (ATP).

The common bond between sleep and seizures is neuronal energy homeostasis. Sleep replenishes glial energy reserves that support neuronal activity during wakefulness. This glial energy resource is accessed when neurons are deprived of the critical nutrients, glucose and oxygen, that are required to generate neuronal mitochondrial ATP.

If nutrient deficiency and energy debt continue unabated, a primitive reflex mechanism, a seizure is activated in a final, desperate attempt to restore neuronal energy homeostasis. A teleological, Darwinian perspective is offered to explain the primordial link between the full tonic-clonic seizure and neuronal energy metabolism, and why this relationship has persisted through the eons. In summary: a seizure starts when normal energy reserve mechanisms fail to meet increased neuronal energy demands and terminates when glucose and oxygen, critical nutrients for the synthesis of mitochondrial ATP, are delivered to deprived neurons by an intense cerebral-hemodynamic response associated with the seizure itself.
\end{abstract}

\section{Introduction}

Seizures were observed and recorded in ancient times and have undoubtedly afflicted human beings since the dawn of our species. Modern medical science has had some limited success in controlling seizures by pharma-chemical suppression of the central nervous system (CNS) and by surgical ablation of the epileptigenic focus in the brain. Progress in molecular biology and genetics has identified various classes of ion channels and neurotransmitters that are major determinants of neuronal excitability and synchronization, the putative "causes" of seizures. However, despite these advances, there is still no satisfactory explanation as to when and why a seizure begins, and when and why a seizure terminates. A clear understanding of these fundamental processes has obvious therapeutic implications.

The search for a unifying concept of seizure onset and termination is supported by evidence from two seemingly disparate sources: sleep physiology, and a teleological theory of the origin of seizures (ictogenesis) [1]. Their common feature is energy homeostasis of the neuron.

\section{Discussion \\ Sleep Physiology}

It has been said that if sleep doesn't have a very important function, it is the biggest mistake that evolution ever made, humans spend one third of their lives asleep, and some animals much more.

Theories of the function(s) of sleep are varied, and remain speculative. One persuasive hypothesis is that sleep restores brain glycogen energy reserves that are depleted by neuronal activity during wakefulness [2].

The energy source in glycogen is adenosine triphosphate (ATP), which, when degraded, forms the molecule adenosine. Adenosine activates the sleep centers in the basal forebrain, and is the major signal controlling EEG slow wave activity through its action on potassium ion channels [3].

The brain requires a great deal of ATP, the coinage of energy exchange, to drive the many functions of the neuron and other cellular components. Although only $2 \%$ of the total body mass, the brain utilizes about $20 \%$ of the entire body's energy expenditure in the resting individual.

However, the brain is dependent upon glucose as the exclusive fuel source to produce ATP and 
upon oxygen to release the energy from its bonds. It has a voracious appetite for oxygen and sugar accounting for $20 \%$ of the total body oxygen consumption and $25 \%$ of total body glucose utilization.

Failure to provide the brain with oxygen and glucose, immediately, continuously and in adequate amounts, can lead to unconsciousness and death within minutes. The brain has evolved an energy reserve strategy to avert this fatal scenario.

We propose that this survival mechanism, when overextended and then restored, accounts, respectively, for the onset and termination of seizures.

\section{What is the brain's energy reserve strategy?}

There are myriad reasons within the circulatory, respiratory and metabolic systems why neurons may become deprived of their critical nutrients. The final common pathway leading to the demise or recovery of the neuron in these conditions depends in large part on the success or failure of compensatory mechanisms to restore neuronal energy homeostasis [4].

When oxygen and carbohydrates are abundant in the blood stream, glial (astrocyte) cells in the brain synthesize glycogen- a long chain polymer of glucose molecules. The glial cells are poised to release the energy from their glycogen stores at a moment's notice if neuronal energy needs are not being met. During sleep, glial glycogen stores are replenished [2].

When the electrical activity in a brain area suddenly increases and/ or local perfusion is inadequate to supply sufficient glucose, glial cells rapidly access their glycogen stores. Energy is released from the third high energy phosphate bond of ATP, and adenosine diphosphate (ADP) is formed. If needed, ADP is further degraded to adenosine monophosphate (AMP) with the subsequent release of the molecule adenosine. High levels of extracellular adenosine are an indication that local energy needs are not being met [5].

Adenosine opens potassium channels in the neuronal membrane. This causes the positively charged potassium ions to move out of the neuron causing the electrical potential across the membrane to become more negative-that is, hyperpolarized. It is now possible for calcium channels to open resulting in a rush of calcium ions into the neuron causing a depolarization of the membrane and the formation of synchronized action potentials. It is these action potentials of billions of neurons sweeping across the brain that are measured as the slow EEG waves characteristic of deep sleep and seizures.

Adenosine also inhibits the cortically projecting basal forebrain arousal system [6], which induces the altered state of consciousness that is characteristic of deep sleep and seizures. The metabolic correlates of sleep and seizures are related to ATP bioenergetics, as evidenced by elevated levels of the metabolite, adenosine, in both conditions. In sleep, to restore glial glycogen energy stores, and, in seizures...to mitigate disturbed neuronal energy homeostasis.

However, in order to advance the concept that a seizure is a remedial response to a neuronal energy-deficient state, it is necessary to reconcile that claim with the visually offensive, psychologically disturbing image of a human in the throes of a full tonic-clonic convulsion.

\section{The paradoxical dual nature of a seizure:}

A seizure can be viewed from two perspectives: events that are occurring simultaneously within the brain (intracerbral), and those external to the brain (extra-cerebral).

\section{Intra-cerebral}

There is considerable evidence in the scientific literature that seizure activity is associated with an intense increase in cerebral perfusion to the focal seizure site.

Weinand and colleagues [7], demonstrated that reduced blood flow to the temporal lobe epilepsy focus was correlated with increased seizure activity and that increased blood flow to the area was associated with decreased seizure activity.
Studies using Functional MRI (fMRI) support these findings [8], Watanabe et al. [9], using Near Infra-Red Spectroscopy (NIRS), showed an increase in blood flow to the focus in $98 \%$ of 32 subjects during drug-induced seizure states.

The purpose of this hemodynamic response, we propose, is to replenish critical nutrients, glucose and oxygen that have been depleted and are needed to restore neuronal energy homeostasis.

\section{Extra-cerebral}

Fay [10] proposed that the visible, neuro-muscular features of a seizure, ranging from an almost imperceptible, transient lapse of awareness and function, to unconsciousness and a full tonic-clinic convulsion, are manifestations of a primitive, protective reflex in response to a life threatening threat to the brain. This unorthodox view is based on the observations that;

1. A seizure can be induced in virtually any vertebrate animal, including every neurologically normal human child and adult,

2. The full tonic-clonic seizure displays stereotypical motor behavior irrespective of its' "cause"

3. Seizures have survived the challenge of eons of Evolution's natural selection process for survival value. Seizures have not become extinct, but are, in fact, a commonly observed phenomenon within the animal kingdom.

Fay proposed, further, that this deep reflex, dormant in all humans, was inherited from our evolutionary forebears, the amphibians.

It evolved as an emergency reflex response to ensure the availability of life-sustaining water, oxygen, salts and electrolytes as primitive creatures struggled to transition from the sea to the land environment. Early life forms embarking on this treacherous journey evolved simple motor patterns concerned with swimming, paddling, and crawling about on the belly, aided by crude legs responding to flexorextensor impulses.

A "defense" pattern in the nervous system, a "seizure", involving tail-twisting and flapping, jerking, and slapping motions ultimately evolved that could return the adventurous explorer to the sea if it wandered beyond the safe harbor of a water hole on land. The life sustaining sea... that would restore oxygen, and vital nutrients to the critically deprived neurons.

A modern, visual analogue is the image of a fish, yanked from the sea, having a frantic "grand mal seizure" on the bottom of the row boat that, on occasion, propels it back into the life sustaining water.

\section{Conclusion}

This Darwinian perspective provides meaning to the otherwise meaningless, widespread distribution of seizures throughout the animal kingdom.

It views ictogenesis as a functional neuronal metabolic disorder that depends on, a) the availability in the environment of critical energy metabolites, oxygen and glucose, b) the ability of the circulatory system to deliver these substances to the deprived neuron, and,

c) the integrity of the NREM energy restorative mechanism.

This energy-centric approach provides a plausible mechanism for the two largest populations affected by seizures- early childhood and the aged- and, a possible explanation for why, in the large group of individuals with genetic defects (channelopathies), seizures occur only intermittently, or are delayed for many years, despite the continued presence of the defect.

Epilepsy is a common medical condition that is currently defined, not by a specific seizure characteristic, but, rather, by any seizure activity that occurs periodically and is, presumably, unprovoked.

The perspective provided in this paper supports the unorthodox view that epilepsy is primarily a metabolic disorder that is manifested, secondarily, by aberrant central nervous system (CNS) function.

Future studies are needed to scrutinize this concept and, if validated, to explore its therapeutic implications. 


\section{References}

1. Fay T. The other side of a fit. Am J Psychiat,1942; 39:196-200.

2. Bennington JH, Heller HC. Restoration of brain energy metabolism as the function of sleep, Progress in Neurobiology, 1995;45:347-360.

3. Dunwiddie TV, Masino SA, The role and regulation of adenosine in the central nervous system. Annu Rev Neurosci, 2001;24:31-55.

4. Takamo T, Tian GF, Peng W, et al. Astrocyte mediated control of cerebral blood flow. Nature Neuroscience, 2006;(9)2:250-267.

5. LLyod HGE, Lindstrom K, Friedholm BB, Intracellular formation and release of adensine from rat hippocampal slices evoked by electrical stimulation or energy depletion, Neurochem Int, 1993;23:173-185.

6. Christie. MA, Bolotuya Y, Microdialysis elevation of adenosine in the basal forebrain produces vigilance impairment in the rat psychomotor vigilance task Sleep, 2008;31(10)1393- 1398.

7. Weinand ME, Labiner DM, Ahern GL. Temporal lobe seizure interhemispheric propagation time depends on nonepileptic cortical cerebral blood flow, Epilepsy Res 2001;44(1):33-39.

8. Salek-Haddadi A, Diego B, Hamandi K, et al., Hemodynamics correlates of epileptiform discharges: an EEG-fMRI study of 63 patients with focal epilepsy. Brain Res, 2006;1088(1):148-166.

9. Watanabe E, Maki A., Kawaguchi F, et al, Noninvasive cerebral blood volume measurement during seizures using multichannel near infrared spectroscopic topography. J Biomed Opt, 2000;5(3):287-290.

10. Pelligra R, Doman G, Ictogenesis: the origin of seizures in humans. A new look at an old theory. Med Hypoth, 2003: 60(1):129-132. 